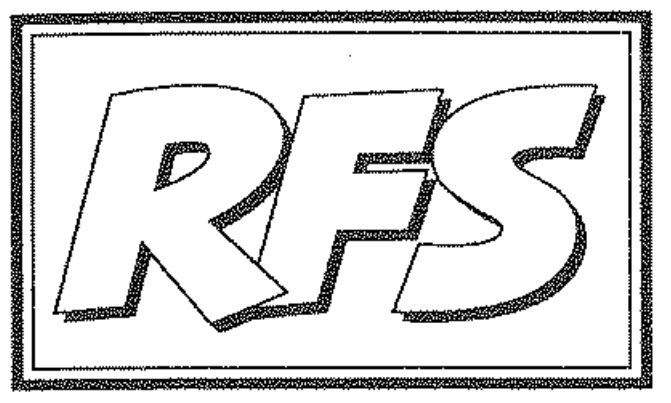

Revista de Fomento Social, $50(1995), 455-471$

\title{
Un instrumento para la promoción de la justicia: 50 años de la Revista de Fomento Social
}

Consejo de Redacción

Con este ejemplar que llega a nuestros lectores, nuestra revista alcanza felizmente el número 200. Doscientos números de una publicación trimestral como la Revista de Fomento Social (RFS) significan 50 años de vida, las bodas de oro. Una larga historia permanente, ininterrumpida, ya que nunca la RFS dejó de acudir a la cita periódica con sus lectores.

En octubre de 1946 aparecía el primer número, acogido cálidamente por el público al que la revista iba especialmente dirigida; esto sirvió de estímulo a la dirección para seguir adelante sin desaliento. Ahora, 50 años después de aquel comienzo, nos sentimos impulsados a hacer balance: pensamos que una mirada retrospectiva nos ayudará a tomar más conciencia de nuestra historia $y$ que así podremos relanzar mejor la RFS hacia el futuro. Y creemos que a nuestros lectores no les resultará superfluo acompañarnos en esa parada de 
reflexión desde el presente hacia el pasado y hacia el porvenir. Al fin y al cabo, ellos son también protagonistas de esta historia y con ellos contamos para diseñar el futuro.

La revista no brotó por generación espontánea; ni fue fruto de una corazonada instantánea; fue, más bien, la culminación de un proceso de deseos y recelos, optimismos y temores, superado todo en ese comienzo de octubre de 1946.

\section{Antecedentes. La revista y su entorno}

Para mejor comprender este medio siglo de historia de la revista, conviene distinguir entre Fomento Social como institución originaria, Fomento Social como revista propiamente dicha y, más recientemente, el denominado grupo Fomento Social.

A) "Fomento Social" como institución

La Institución Fomento Social fue el fruto del tesón y de la visión certera del Padre Sisinio Nevares (1). Nevares no fue el primer jesuita español que actuó en el campo social, ni el único en su tiempo, aun cuando hay que reconocer que no eran numerosas los jesuitas que en aquella época dedicaban su actividad apostólica al ámbito social. Nevares fue un autodidacta, como tantos otros. Sin entrar en el análisis de otros campos, en el del apostolado con sus varias formas, se repite el caso-frecuente en la historia de la Compañía-del hombre dinámico, profético, sintesis de idea y acción, hasta genial en su valer doctrinal y su empuje físico, que concibe un plan, lo plasma en posibles estructuras en su mente y se lanza a realizarlo solo. Nevares desplegó una actividad frenética que, tomando como centro de irradiación la Casa Social de Valladolid, anda y desanda los caminos de la geografia de España.

Aun cuando esencialmente era un hombre de acción, Nevares comprendió la importancia de un Centro de estudios sociales y de difusion a escala nacional. Vio la necesidad de trabajar en equipo, buscando la fuerza de una organización que no confiara todo a la fortuna de una sola persona. Pensó mucho el proyecto

(1) Cfr. GiNER, C. (1987), "Sisinio Nevares (1878-1946): un doctrinario del catolicismo social español”. RFS, n" 5, pp. 313-327. 
y lo sometió a sus superiores de Roma. Quería reunir en Madrid a especialistas que consagraran toda su actividad a esta idea, con archivos y biblioteca especializada, como imprescindibles instrumentos de trabajo; habia puestolos ojos, como fuente de inspiración, en la Action Populaire de los jesuitas franceses. El entonces general de la Compañia, el polaco P. Ledokowski, valoró la importancia de la obra, la apoyó moral y económicamente, y en 1926 nacía Fomento Social.

La institución se desarrolló bajo la dirección de Nevares hasta 1935. Entre los primeros colaboradores hubo hombres de acción, conocedores del mundo obrero español y de los movimientos sociales extranjeros. En los seguidores inmediatos de este primer equipo hubo menor preparación especializada que en los de la última hornada; éstos últimos con preparación académica y una orientación socioeconómica especializada. Su actuación atiende más a la problemática de una sociedad evolucionada; cultiva más el campo de la reflexión, en grupos de estudio, que alimentan la revista, sin olvidar la cátedra y las conferencias al gran público.

La actuación del primer equipo que se instala en la sede de la "Casa de Escritores" de los jesuitas de Madrid, en la calle Pablo Aranda-fue diversa: conferencias a distinto público, cursillos de orientación social y la redacción y publicación de los primeros libros de la "Biblioteca Fomento Social" especializada. Sin revista propia, aquel equipo colaboró en la más antigua revista jesuítica "Razón y Fe", divulgando además el Pensamiento Social de la Iglesia con un "Servicio de Prensa", que ponían a disposición de muchos diarios de España.

B) Nacimiento de la revista

Como revista, "Fomento Social" nace en octubre de 1946, bajo la dirección del P. Joaquín Azpiazu (2). En el pórtico se dice que

“...quiere ser una revista de Sociología y de Moral económica, sin aires y sin apariencia de una revista técnica y económica, o de una revista de

(2) Véanse sobre la personalidad de Azpiazu (1887 1953), su trayectoria, sus escritos y su significado social varios artículos en el número 31 de la RFS, correspondiente al año 1953. 
Sociología pura. Quiere, sin embargo, estudiar los hechos, teorías y doctrinas económicas y sociales, tanto de España como del extranjero, para enfocarlos a la luz de la moral cristiana, exaltándolos si lo merecen, opresentándolos al mundo tal como deberían ser si de ella se desvian...".

"Pretende Fomento Social, difundiendo en sus páginas la doctrina de la Iglesia, modernizar las relaciones económico-sociales de la Humanidad, ayudando en su pequeñez a la labor ingente de los Pontifices de Roma que, sin meterse a economistas ni sociólogos puros, iluminan los temas económico-sociales con la luz perenne de la doctrina católica; oponen a la critica negativa la doctrina positiva y salvadora".

En el editorial primero se justifica el porqué de su aparición. "Es la hora de la Iglesia", se decía. Había que encauzar las ideas revolucionarias mediante la aplicación de la moral; era preciso avanzar en la recta comprensión de las ansiadas libertades; había que colaborar a la formación del sentido social, mirando al bien común, que vale tanto como saber de justicia social; todo ello "movidos por la caridad social, que lleve a la conmoción social ante el necesitado". Queda así comprometida la revista con un claro programa ante el público. ¿Lo ha cumplido?

\section{C) El "Grupo Fomento Social"}

Por último, recientemente, en estrecha relación con la revista y como apoyo a la misma, nació en 1987 el llamado "Grupo Fomento Social" (jesuitas españoles en las ciencias sociales y en la acción social) cuyos fines estatutarios son los siguientes:

"Procurar periódicamente el contacto y los intercambios de punto de vista, y favorecer la cooperación entre jesuitas residentes habitualmente en España y dedicados, bien al estudio de la sociedady de los problemas sociales, bien a la acción social directa y comprometida.

Favorecer las relaciones con otras organizaciones similares de dentro o de fuera de la Compañia, y con los jesuitas de dedicación paralela en otras partes del mundo". 
Desde 1987 este grupo, formado casi exclusivamente por jesuitas, se viene reuniendo una vez al año. A lo largo de sus encuentros ha reflexionado sobre temas tales como: El paro, El capitalismo democratico, Nueva pobrezaMarginación social, Tras la crisis del socialismo real, El desequilibrio NorteSur, Posición de la Compañia ante el problema de la justicia (ante la celebración de la Congregación General 34), Viabilidad de los valores de la justicia en el sistema económico vigente, El futuro del sindicalismo.

Normalmente, nuestra revista se ha hecho eco, en sendas crónicas, de cada una de las reuniones de este grupo. Por otro lado un buen número de las ponencias y comunicaciones presentadas a esas jornadas de estudio y reflexión han sido publicadas en la propia revista (3).

\section{Il. Cincuenta años de historia de la revista (4)}

Centrándonos en la RFS propiamente dicha, podemos distinguir varias etapas en su extensa trayectoria, definidas -comonopodia sermenos-tantopor modificaciones "endógenas", debidas a cambios en la evolución de la mentalidady la praxis de la Compañia de Jesús, en las personas y equipos de trabajo (5), como por cambios "exógenos" producidos en el entorno nacional, sobre todo, político. Ni que decir tiene que esos dos ámbitos han estado siempre en estrecha relación. En cualquier caso se trata, como es natural, de una clasificación que siempre tiene algo de convencional. Recorriendo estas etapas, vemos reflejarse en las páginas de la revista la evolución tanto de la sociedad española, como del pensamiento social cristiano; la selección de temas, los acentos y los subrayados, así como las corrientes de pensamiento que se expresan en los juicios y las propuestas, son un fiel reflejo de lo que ha

(3) Los secretarios de este grupo, encargados de la coordinación y organización de las jornadas anuales, han sido sucesivamente Javier Gorosquieta S.J. (1987-1990: a la sazon, también director de la revista); Jaime Loring S.J. (1991-1994) y José Juan Romero S.J. (a partir de 1995).

(4) Nuestros suscriptores recibirán precisamente en los próximos meses los indices correspondientes a este medio siglo de la RFS que actualmente estamos confeccionando.

(5) Recordamos la lista de los directores: Joaquín Azpiazu S.J. (1946-1953); Manuel Marina S.J. (1954-1958); Florentino del Valle S.J. (1958-1970); Victorino Ortega S.J. (1970-1976 y 1981-1987); Javier Gorosquieta S. J. (1977-1981 y 1987-1990); Alfonso C. Morales (19911995). 
sido la problemática social de España, de la evolución del pensamiento de la Compañiay, de alguna manera, de la lglesia española sobre los temas sociales, económicos y políticos. La historia de esa evolución no podrá escribirse sin acudir a las páginas de la RFS.

A) Los primeros años (1946-1953: el Padre Azpiazu). Especial énfasis en las cuestiones laborales

La primera etapa va desde su fundación hasta 1953. Esta primera fase estä muy marcada por la impronta de su fundador y primer director, Joaquín Azpiazu. Gran moralista y sociólogo, buen conocedor del Derecho, su esfuerzo se centró especialmente en la ética empresarial y en el estudio del estado corporativo. No sólo sus trabajos publicados en la revista durante esos años, sino también sus dos reconocidos libros, La Moral del Hombre de Negocios y El Estado Corporativo, avalan esa afirmación. Su actitud no fue revolucionaria o de oposición, sino reformista dentro del sistema económico y político. Azpiazu había sido, por ejemplo, uno de los principales redactores del Fuero del Trabajo de 1938.

La atención de la revista se centraba en los graves problemas económicos, sociales, laborales de nuestro pais, sumido en un notable aislamiento politico y en una marcada autarquía económica. En estos primeros años, la influencia del citado benemérito P. Azpiazu fue predominante, pues se cuentan hasta 55 artículos suyos en 8 años, con firma opseudónimo; ahora bien, el centro de sus preocupaciones, partiendo de una buena formación jurídica y económica, era la moral profesional económica.

En estos años la llamada "cuestión social" está predominantemente centrada en las relaciones capital trabajo y en los problemas de indole laboral. En efecto, en cuanto a los temas tratados destacan con luz propia los problemas laborales y los temas relativos a la ética; sin embargo, se habla muy poco-casi nada-del sindicalismo y de los problemas conexos... Por último aparecen con frecuencia colaboraciones sobre la doctrina social de la Iglesia en sentido estricto. En general, la inmensa mayoría de los artículos se refieren a España, siendo escasos los que hacen referencia al ámbito internacional.

En este mismo período de 8 años, además de Azpiazu, otras dos firmas aparecen de forma muy predominante al pie de los artículos de la revista: 
Martin Brugarola (1908-1988); y Florentino del Valle. Con Azpiazu, son autores de casi la mitad de los artículos del periodo. Con menor frecuencia, aparecen también los nombres de Aresio González de Vega, Angelo Perego, Angel Torres Calvo, Fermín de Urmeneta etc.

B) Años de buenas relaciones con el régimen (1954-1962)

Con la muerte de Azpiazu en 1953 se abre una nueva era para la RFS. Manuel Marina (1895-1987) le sustituye como director. Eran los tiempos de feliz matrimonio entre la Iglesia y el régimen franquista surgido de la guerra civil. No se cuestiona este último, y los nuevos responsables de la revista procuran contribuir a lubrificar desde dentro, y con una aspiración de mayor justicia y equidad, los engranajes políticos, económicos, sociales, laborales del sistema establecido. Todo ello desde la doctrina social de la Iglesia: se reproducen, como argumentos de autoridad, y comentan abundantemente los textos formales de esta última. También se hizo un esfuerzo notable por informar sobre la existencia y la naturaleza de instituciones sociales modernas, nacionales e internacionales tales como las Semanas Sociales, los movimientos obreros cristianos, asociaciones católicas de empresarios, sindicatos, congresos nacionales e internacionales etc. Se desarrolló, asimismo, una asesoría laboral de corte técnico.

Es verdad que en esta etapa hubouna fuerte crítica al sindicatovertical, pero fue en el sentido de querer darle una mayor autonomía respecto de la Administración y del Ejecutivo, es decir, más claramente, en la linea de las aspiraciones del nacionalsindicalismo y de acuerdo con la doctrina social de la Iglesia, tal como había sido formulada en la encíclica "Quadragesimo anno" en 1931.

En este periodo, la revista presentaba sistemáticamente sendas crónicas: "Crónica internacional" (firmada por Martín Brugarola (6)) y "Crónica social de España", habitualmente redactada por Florentino del Valle. Las primeras eran una especia de "observatorio" de la realidad mundial con una perspectiva ética y cristiana; consistian en una síntesis (en 6-8 páginas) de los

(6) Cfr. Consejo de Redacción (1989), "In memoriam": P. Martín Brugarola i Mas (1908" 1988 )". RFS, n" 1773 , pp. $5-6$. 
acontecimientos más relevantes del entorno internacional. Por cierto que en el decenio 1956-1965 se concentra más de la tercera parte de todas las colaboraciones que sobre el tema internacional hemos contabilizado en los 50 años de la publicación. Por su parte, la "Crónica social de España" estaba consagrada a la realidad nacional sobre todo en su vertiente empresarial/ laborallsindical. También fueron frecuentes, aunque menos numerosas y sistemáticas, las crónicas "legislativa" y sobre el "magisterio eclesiástico".

Ahora bien, en términos generales, pese a que en esta segunda etapa aumenta sensiblemente en la revista el tratamiento de los temas internacionales, la problemática del tercer mundo es muy escasamente abordada. Sobre el entorno nacional siguen apareciendo los temas laborales y sindicales y un número significativo de artículos referidos al mundo de la empresa. Sin duda, la doctrina social de la lglesia y el magisterio aparecen también con frecuencia; el ámbito nacional sigue siendo predominante.

En lo que concierne a los colaboradores más frecuentes, ya desaparecido Azpiazu, destacan en los 9 años de esta época las firmas de los dos beneméritos jesuitas citados: Florentino del Valle y Martín Brugarola; en menor medida encontramos también frecuentes colaboraciones de Manuel Marina y de Angel Torres Calvo.

C) Años de conflictos con el franquismo: (1963-1974)

En 1963 se incorpora al Instituto Fomento Social y a su revista un equipo de jôvenes jesuitas. En la Iglesia universal son los años del Concilio y su entusiasta y conflictiva aplicación. En la Iglesia española, incluidos los sacerdotes y los militantes cristianos, va creciendo una corriente de crítica y oposición, más o menos clandestina, más o menos entre líneas, al régimen establecido y a sus instituciones políticas, sindicales, económicas, sociales. En esta nueva etapa, que va hasta la muerte de Franco en 1975, la RFS se va sumando a estas actitudes críticas. Propugna sobre todo presionar hacia la reforma de las estructuras desde planteamientos y análisis técnicos, inspirándose en el pensamiento social cristiano. No siempre fue fácil hacerlo, frente a la rígida censura que tantas veces devolvió los textos cercenados por el boligrafo rojo. Pero hubo que aprender el sutilestilo de comunicar entre lineas.

Se observa en estos años un más acentuado repliegue sobre los temas 
nacionales. La agitación de la vida política y social española y los numerosos cambios socio-politicos necesarios llevaron a los responsables de la publicación a centrar su atención de forma casi exclusiva en la problemática nacional. Se puede quizás afirmar que la crisis económica mundial -materializada de alguna manera en la crisis monetaria de los primeros años 70 y el estallido de la guerra del Yon-Kippur a finales de 1973 con sus traumáticas consecuencias sobre el precio del petróleo- cogió algo desprevenida a la revista; como a todo el país, por cierto... Sin embargo inspiró algunos muy tempranos y significativos articulos de Javier Gorosquieta y Adolfo Rodero (7).

En esta época (doce años en total) Florentino del Valle continúa escribiendo con frecuencia hasta 1970 y aparecen como firmas predominantes Javier Gorosquieta y Victorino Ortega (8). También aparecen, aunque en menor medida, las firmas de Victor Manuel Arbeloa, Francisco Belda, Eugenio Recio y Gonzalo Higuera; el benemérito P. Brugarola aún aparece de forma más esporádica.

D) En un nuevo contexto sociopolítico (1975-1990)

Una nueva fase comienza en noviembre de 1975. La muerte de Franco, la transición política y el proceso de normalización democrática marcan profundamente este período. La revista se incorpora al movimiento que demanda la implantación de las libertades políticas, económicas, sindicales. A partir de la llegada formal de la democracia con la Constitución de 1978, la RFS procura hacer una tarea de acompañamiento del proceso, orientando siempre hacia estructuras y soluciones más justas, solidarias, fraternales. La larga época de gobierno socialista también ha quedado reflejada en sus páginas de una u otra forma. En esa tarea de acompañamiento critico, desde una perspectiva ética y cristiana, se ha mantenido hasta ahora.

(7) En el n 111 , de 1973; ambos sobre la crisis del Sistema Monetario Internacional. Mucho más tardía es, por ejemplo, la serie de 4 artículos de F. Martínez Galdeano sobre la evolución del mercado del petróleo, sobre todo con motivo de la gran segunda crisis energética $\left(n^{\circ} 137\right.$ a 140 , en $\left.1980 ; \mathrm{n}^{2} 144,1981 ; \mathrm{n}^{\circ} 145,1982\right)$.

(8) Victorino Ortega (1929-1987) falleció prematuramente siendo director de la revista. Cfr. Gorosquieta, J. (1987), "El pensamiento social de Victorino Ortega". RFS, n² 166, pp. 119-132. 
Sin abandonar la atención preferente a los temas sociales, políticos y económicos de ámbito nacional, a partir de 1980 aumentan de manera significativa los enfoques globales, mundiales; espigando a lo largo de los números de la revista, nos encontramos con aportaciones realmente valiosas sobre la crisis mundial, la problemática Norte-Sur, el desarrollo etc. Curiosamente, la atención a Latinoamérica, que es muy escasa (prácticamente inexistente) en el decenio 1976-1985, aumenta de forma considerable a partir de este último año.

En los 16 años de este periodo de instauración y consolidación de la democracia, destacan por su frecuencia de aparición las firmas de Javier Gorosquieta y Victorino Ortega, seguidos por Gonzalo Higuera, Matías García Gómez, Enrique Menéndez Ureña, Francisco Gómez Camacho, Ildefonso Camacho, Manuel Alcalá y F. Martínez Galdeano.

E) En la nueva sede (Córdoba: 1991-1995)

En 1991 se inicia la última fase, hasta el momento, de la publicación. No se trata de un cambio de contenidos o de linea editorial; ambas siguen en continuidad con la etapa anterior. Se ha producido simplemente un traslado institucional. Desaparecido el equipo del Instituto Fomento Social de Madrid por la jubilación de algunos de sus miembros y por diversos destinos de otros, se hace cargo de la empresa de la revista la obra universitaria de la Compañía en Córdoba, el INSA-ETEA, que comprende entre otros centros la Facultad de Ciencias Económicas y Empresariales adscrita a la Universidad pública de Córdoba. Se trata de una continuidad y de unas mismas preocupaciones que en la etapa anterior. Asi se manifiesta en el artículo editorial del número de enero-marzo de 1991; así aparece en el contenido de los números de estos últimos cinco años. No en vano varios profesores de INSA-ETEA habían venido colaborando desde antiguo con la RFS.

En esta época, un periodo de 5 años más corto que los anteriores, no hay ningún autor predominante pero sí se constata una diversificación de la nómina de colaboradores, con presencia muy mayoritaria de seglares. Además de ampliarse el abanico de colaboradores, aumenta la nómina de los "del sur", y también parece incrementarse la interdisciplinariedad de los temas tratados, con mayor incidencia de los enfoques juridicos y culturales, tan decisivos en las 
preocupaciones de la revista por una evolución hacia una sociedad más justa. También puede constatarse que de un centenar de artículos, algo más del $25 \%$, están firmados por profesores de ETEA; se trata, por tanto, de una presencia significativa pero "discreta"... En todo caso, se trata de un periodo todavía corto y, por otro lado, los miembros del actual Consejo de Redacción, que redactamos estas líneas, no somos quizás los más indicados para hacer un juicio de valor sobre estos últimos cinco años. Al ser los más recientes, están seguramente más presentes en la memoria de nuestros amigos lectores, y es a ellos a quienes corresponde evaluar el proceso seguido.

F) Lo que cambia, lo que permanece

Es difícil hacer una síntesis de estas cinco décadas, Quizás los lectores que han sido fieles durante muchos años a la RFS serian los mejor preparados para hacerla. Desde nuestro observatorio -inevitablemente parcial-percibimos cómo la RFS refleja con bastante exactitud la evolución de la sociedad española y de la Iglesia. Respecto a esta última, el concilio Vaticano II marca un cambio de rumbo decisivo, que tiene su eco inmediato en los católicos españoles y en las relaciones de nuestra Iglesia con la sociedady con el estado. Todo esto encuentra una innegable resonancia en la RFS, marcado además por la evolución de la Compañía de Jesús, de su mentalidad y de la forma de entender su misión en el mundo moderno.

Nos atreveríamos todavía a concretar estos cambios en las siguientes líneas: paulatinamente se va reduciendo el tono clerical de los primeros tiempos, tanto en la temática como en los colaboradores; se gana en apertura, en cuanto al abanico de problemas y a los destinatarios (más abierta a lectores no creyentes); se avanza en interdisciplinariedad; se hace más "universitaria".

Peroen medio de tantos cambios hay también unas constantes, unas fidelidades, que podriamos enumerar así: una permanente preocupación ética; un énfasisen la doctrina social de la Iglesia; una constante presencia como órgano de opinión comprometida y crítica (hasta 248 artículos editoriales se pueden contar en sus 200 números, más de uno por número); un esfuerzo permanente por el rigor en el tratamiento de los temas, pero evitando siempre el excesivo tecnicismo para poder estar al alcance del lector culto aunque no especializado en los temas abordados; un talante crítico, pero constructivo, creativo y abierto al cambio. 


\section{IIl. Mirando al futuro}

Sin duda que ese pasado, del que hemos ofrecido una panorámica amplia, es historia. Pero, en este caso, es nuestra historia, la base inexcusable sobre la que tenemos que seguir avanzando. Si el número 200 de nuestra revista ha sido una buena oportunidad para hacer balance, éste no tendría sentido si no es con vistas al futuro que proyectamos. Por eso en las páginas que siguen queremos reflexionar sobre ese futuro que se abre ante nosotros: qué pretendemos con nuestra revista, cómo desarrollaremos esos objetivos, con qué base humana contamos, a quiénes desearíamos llegar.

A) Qué queremos que sea la RFS (objetivos)

La RFS nació por iniciativa de la Compañia de Jesús. A lo largo de estos 50 años ella ha querido mantenerla, aun cuando no hayan faltado las dificultades y se haya tropezado muchas veces con la escasez de recursos para llevarla adelante. Esta voluntad firme de seguir se apoya en el convencimiento de que continúa siendo un instrumento válido al servicio de su misión.

Durante los 50 años de vida de la RFS han sido muchos y decisivos los acontecimientos, sin excluir los del ámbito eclesial. En concreto, el Concilio VaticanoII propició un replanteamiento sustancial de la forma en que la Iglesia se situaba en la sociedad. En los 30 años que nos separan ya del Concilio la Iglesia ha buscado, no sin vacilaciones y resistencias, nuevas vías de presencia, menos jerárquicas y más cercanas, menos doctrinales (ahistóricas) y más condicionadas por la provisionalidad de lo histórico.

A estos cambios también ha querido responder la Compañía de Jesús revisando sus actividades y sus instituciones y actualizando el modo de entender su misión. Ahi están las cuatro últimas Congregaciones Generales (la primera de ellas, recién concluido el Concilio; la última, en los primeros meses de 1995) como hitos de un proceso tampoco exento de dificultades internas y externas, a través del cual se procedió a una reformulación actualizada de la misión primero, y posteriormente a evaluar dicha reformulación y a profundizar en su alcance y consecuencias.

Con tales presupuestos, nos parece que este 50 aniversario nos ofrece una ocasión inmejorable para reformular también nosotros los objetivos que 
marcan nuestra tarea:

19) La orientación última de la RFS está en función del servicio de la fe y promoción de la justicia, tal como ha sido asumido en las Congregaciones citadas: la perspectiva cristiana es, pues, determinante; pero queremos que ella se proyecte sobre los innumerables y variados ámbitos donde está en juego la construcción de un mundo más justo y más humano.

$\left.2^{\circ}\right)$ La promoción de la justicia nos lleva a interesarnos en concreto por todos los problemas relacionados con la vida social, económica, politica y cultural, en su doble vertiente nacional y mundial. La preocupación social, que tanto marcó a la RFS desde sus comienzos con una atención preferente a lo socioeconómico, la reasumimos hoy matizándola en una doble dirección: somos más conscientes de los vínculos estrechos que liga a lo socioeconómico con lo político y lo cultural; estamos convencidos de que nuestros problemas sólo pueden ser entendidos en el marco más amplio de la sociedad planetaria, con sus dramáticas discriminaciones y con su anhelo cada vez más perceptible de unidad. Para todo ello es imprescindible la mediación de las ciencias sociales: al recurrir a ellas nos interesa garantizar el mayor rigor cientifico, pero sin dejar por ello de prestar atención a los valores inevitablemente en juego en todo discurso cientifico (dimensión ética).

39) La RFS quiere ser instrumento de divulgación del pensamiento social cristiano; pero quiere ser también un lugar de encuentro y de diálogo con otras cosmovisiones que conviven en la cultura moderna. En un contexto tan pluralista como el nuestro, la fe y cultura cristianas no pueden encerrarse en sus propios planteamientos y desentenderse de los de otros: la realidad que nos rodea nos invita, más bien, a unir esfuerzos para hacer frente a la complejidad de los problemas que tenemos delante; y la coherencia de la fe corrobora esta orientación.

$\left.4^{9}\right)$ Por úlimo la localización concreta de la $R F S$, que reside desde 1991 en una institución universitaria consagrada desde hace ya más de 30 años al mundo de la empresa, es un acicate para prestar una atención especial a esta realidad. La empresa es una institución clave en la vertebración de la sociedad, creadora de riqueza y de empleo; de su promoción, existencia y buen funcionamiento depende en gran medida el bienestar de la sociedad. Desde estas paginas queremos promover un visión coherente y no parcial de la empresa: una comunidad de personas, aunque a veces las enfrenten objetivos 
diferentes, con unos objetivos económicos pero también con una inexcusable función social.

B) De qué medios nos valemos

En una revista se pueden dar cita materiales muy diversos. Su género también puede ser variado. Nosotros utilizamos cinco tipos de materiales que describimos brevemente a continuación.

Queremos que todo número de la RFS se abra con un artículo editorial. Es un trabajo del Consejo de Redacción y que se publica bajo su responsabilidad solidaria, aunque muchas veces hayan colaborado en él, de forma más que desinteresada, otras personas. Dicho articulo editorial suele ser de cierta extensión, ya que sólo asi es posible entrar con cierta profundidad en los temas que abordamos, normalmente seleccionados de entre las inquietudes dominantes del momento. El carácter trimestral de la RFS no nos permite abordar hechos muy puntuales y pasajeros, sino cuestiones de más alcance, pero que son decisivas para interpretar el día a día de lo que va aconteciendo. En la selección de los temas y en el tratamiento que hacemos de ellos queremos dejar constancia de cómo la RFS toma posición ante el mundo que nos rodea: reflejaria, por asi decirlo, la postura oficial de la revista.

Un fruto de este esfuerzo de los cinco últimos años ha sido el volumen Sociedad y economía en los años 90. Reflexiones de la RFS, que se acaba de publicar para conmemorar estos 50 años de la RFS, y donde hemos recogido y actualizado los artículos editoriales aparecidos en ese tiempo.

Los estudios y las notas constituyen por lo general la parte más extensa de cada número. Intervienen en ellos diferentes colaboradores, a los que nos referiremos en seguida. A veces son trabajos solicitados por nosotros; otras veces nos llegan espontáneamente: en ambos casos son seleccionados atendiendo a la adecuación de su tema a los objetivos de la revista y al rigor con que están elaborados. Esta exigencia de rigor no es menor en la notas: esta denominación obedece más bien a su carácter más coyuntural, a su mayor brevedad, a la ocasión para la que fue compuesto el trabajo, a la ausencia de bibliografia, etc.

Nos agrada también ofrecer a nuestros lectores crónicas de acontecimientos o celebraciones que tienen alguna relación con nuestra revista, con su temática 
o con sus colaboradores más estables. Son testimonios de iniciativas que conectan con la línea de la RFS y de las que creemos oportuno dejar constancia ante nuestros lectores.

La sección de documenios ha sido poco frecuentada en estos úlimos años. $Y$ no por falta de ellos. Precisamente es la abundancia de documentos que se publican la que nos movía a seleccionar aquéllos que pensamos de mayor interés para nuestros lectores y que, por otra parte, son de dificil acceso al público en general.

Si abundantes son los documentos de todo tipo que ven la laz en este tiempo, ¿qué decir de la producción bibliográfica en general? El apartado bibliográfico (recensiones y reseñas) pretende extender la labor de selección también a este poblado terreno. No queremos que el número de recensiones sea cuantioso: preferiríamos escoger aquellos libros que juzgamos de mayor interés para nuestros lectores ofreciéndoles una presentación detallada de cada uno y el juicio critico correspondiente. Reconocemos que en esta sección tenemos que mejorar mucho respecto a lo que estamos ofreciendo hasta el momento.

C) Con qué base humana contamos

El mantener viva una revista, y el hacerlo con un nivel de calidad aceptable, exige contar con personas capacitadas y dispuestas a dedicar parte de su tiempo a esta tarea. Los colaboradores de la RFS son numerosos. Podemos agruparlos en tres círculos.

El primer círculo, el más cercano a la revista, lo constituye su Consejo de Redacción y el grupo de colaboradores especiales cuyos nombres aparecen en la contraportada. No somos nosotros los más idóneos para enjuiciar nuestra propia labor. Otros lo hacen sin duda: a veces nos envian sus críticas o sugerencias, aunque no con la frecuencia con que nos gustaría. isería inoportuno aprovechar estas lineas para invitar a todo el que lee (u hojea) nuestra revista a manifestarnos su parecer sobre lo que encuentra en ella?

Del Consejo de Redacción depende directamente, como ya se ha dicho, el contenido de los artículos editoriales y la selección de las demás colaboraciones. En esta selección respetamos un cierto pluralismo, aunque dentro de la orientación global de la revista. Para desarrollar adecuadamente las tareas de la revista, ETEA nos proporciona el imprescindible respaldo económico, la 
labor de secretaria y la infraesiructura necesaria.

Un segundo círculo de colaboradores lo constituyen los profesores de la Facultad y de la Escuela de Ciencias Económicas y Empresariales de ETEA (Córdoba), donde tiene su sede la RFS. Deseamos que sean ellos los primeros que vean en la RFS algo propio, una plataforma donde dar cauce a sus esfuerzos y difusión a sus trabajos.

El tercer círculo, más amplio, es el de nuestros colaboradores externos. Son muchos. Algunos están unidos a nosotros por vínculos más estrechos que el de una mera colaboración esporádica. Dentro de los límites físicos de una revista como la nuestra, nos gustaría que todos encontraran un hueco en ella: eso nos enriquece y enriquece a nuestros lectores. Naturalmente damos prioridad a los trabajos solicitados sobre los de aquéllos a los que no hemos pedido colaboración. Pero somos exigentes en cuanto a calidad con unos y otros (también con los del circulo anterior e incluso, creemos, con nosotros mismos): por eso no nos resistimos en ocasiones a pedir que se corrijan omejoren algunos aspectos de los originales. Rechazar colaboraciones (que también hay que hacerlo a veces) o criticarlas es incómodo a primera vista: pero a la larga es condición para la calidad de la revista; además sirve para establecer un diálogo fecundo entre los autores y el Consejo de Redacción.

D) A quiénes queremos dirigirnos

Naturalmente tras esta última pregunta no se esconde la pretensión de excluir a nadie. Pero pensamos que cada revista tiene su público, en función de su temática y de su orientación.

Entre nuestros destinatarios fijos se encuentran buen número de centros universitarios y otras instituciones tanto privadas como organismos publicos (ministerios, consejerias etc.), agentes sociales (sindicatos, etc.) que se mueven en temas afines a los nuestros. Muchas de esas instituciones nos envian sus publicaciones en régimen de intercambio. También por esa vía se refuerza la colaboración y el diálogo intelectual.

En cuanto a los lectores (y suscriptores) particulares, nos gustaría que fueran personas que muestran inquietud ante los problemas sociales, políticos, económicos y culturales de nuestro mundo, y que están en contacto con ellos desde una determinada actividad profesional, vivida crítica y responsablemen- 
te. Si desde esa óptica sintonizan con nuestra preocupación de promoción de la justicia, entonces nuestra revista les suministrará materiales que alimenten su reflexión y su acción. No pensamos que nuestra revista pueda interesar sólo a lectores creyentes: el tono que utilizamos, que nunca oculta nuestra orientam ción de fondo, quiere estar abierto a toda colaboración que se apoye en una voluntad decidida de búsqueda permanente de la verdad.

Entre tantas publicaciones como ahogan el mercado, el darse a conocer resulta dificil y costoso. Sentiríamos que personas que estarían interesadas en la RFS no la conocieran aún porque no hemos sabido llegar hasta ellas. Por eso también invitamos a nuestros colaboradores y lectores a difundirla entre quienes podrían beneficiarse de lo que publicamos.

Esta invitación a convertirse en difusores de la RFS no la consideramos fuera de lugar. Es cierto que la comunicación a través de las páginas impresas es fría e impersonal. Pero el contacto continuado, aunque sea por esa vía, crea vinculos y va tejiendo una red que a la larga toma las dimensiones y las características de una gran familia.

A nosotros sólo nos resta, para terminar, expresar este deseo y nuestra confianza de que se vaya haciendo realidad: que la RFS sea como el centro de gravedad de una extensa familia de colaboradores y lectores unidos por el ideal de trabajar, desde el puesto que cada uno ocupa en la sociedad, por un mundo más humano y justo, en definitiva más acorde con ese modelo de fraternidad que es el Reino que Dios nos tiene prometido pero que nosotros vamos construyendo día a día en la historia. 